\title{
The Schur-Convexity of the Generalized Muirhead-Heronian Means
}

\author{
Yong-Ping Deng, ${ }^{1}$ Shan-He Wu, ${ }^{1}$ Yu-Ming Chu, ${ }^{2}$ and Deng $\mathrm{He}^{3}$ \\ ${ }^{1}$ Department of Mathematics and Computer Science, Longyan University, Longyan, Fujian 364012, China \\ ${ }^{2}$ School of Mathematics and Computation Science, Hunan City University, Yiyang, Hunan 413000, China \\ ${ }^{3}$ Gangtou Middle School, Fuqing, Fujian 350317, China
}

Correspondence should be addressed to Yu-Ming Chu; chuyuming2005@126.com

Received 16 June 2014; Accepted 15 August 2014; Published 27 August 2014

Academic Editor: Giovanni Anello

Copyright (C) 2014 Yong-Ping Deng et al. This is an open access article distributed under the Creative Commons Attribution License, which permits unrestricted use, distribution, and reproduction in any medium, provided the original work is properly cited.

We give a unified generalization of the generalized Muirhead means and the generalized Heronian means involving three parameters. The Schur-convexity of the generalized Muirhead-Heronian means is investigated. Our main result implies the sufficient conditions of the Schur-convexity of the generalized Heronian means and the generalized Muirhead means.

\section{Introduction}

In what follows, we denote the set of real numbers by $\mathbb{R}$, the set of nonnegative real numbers by $\mathbb{R}_{+}$, and the set of positive real numbers by $\mathbb{R}_{++}$.

Let $(x, y) \in \mathbb{R}_{++}^{2}$; the classical Heronian means is defined by (see [1])

$$
H_{e}(x, y)=\frac{x+\sqrt{x y}+y}{3} .
$$

In 1999, Mao [2] gave the definition of dual Heronian means; that is,

$$
\widetilde{H}_{e}(x, y)=\frac{x+4 \sqrt{x y}+y}{6} .
$$

In 2001, Janous [3] considered the unified generalization of Heronian means $H_{e}(x, y)$ and $\widetilde{H}_{e}(x, y)$ and presented a weighted generalization of the above-mentioned Heroniantype means, as follows:

$$
H_{w}(x, y)= \begin{cases}\frac{x+w \sqrt{x y}+y}{w+2}, & 0 \leq w<\infty, \\ \sqrt{x y}, & w=\infty .\end{cases}
$$

Jia and Cao [4] investigated the exponential generalization of Heronian means

$$
H_{p}(x, y)= \begin{cases}\left(\frac{x^{p}+(x y)^{p / 2}+y^{p}}{3}\right)^{1 / p}, & p \neq 0, \\ \sqrt{x y}, & p=0,\end{cases}
$$

and they established some related inequalities. The monotonicity and Schur-convexity of the Heronian means $H_{p}(x, y)$ were discussed by Li et al. in [5].

Shi et al. [6] discussed the Schur-convexity of a further generalization of the Heronian means given by

$$
H_{p, w}(x, y)= \begin{cases}\left(\frac{x^{p}+w(x y)^{p / 2}+y^{p}}{w+2}\right)^{1 / p}, & p \neq 0, \\ \sqrt{x y}, & p=0,\end{cases}
$$

and they obtained a significant result asserted by Theorem A below. 
Theorem A. For fixed $(p, w) \in \mathbb{R}^{2}$,

(1) if $(p, w) \in\{(p, w) \mid p \geq 2,0 \leq w \leq 2\}$, then $H_{p, w}(x, y)$ is Schur-convex for $(x, y) \in \mathbb{R}_{+}^{2}$.

(2) If $(p, w) \in\{(p, w) \mid p \leq 1, w \geq 0\} \cup\{(p, w) \mid 1<$ $p \leq 3 / 2, w \geq 1\} \cup\{(p, w) \mid 3 / 2<p \leq 2, w \geq 2\}$, then $H_{p, w}(x, y)$ is Schur-concave for $(x, y) \in \mathbb{R}_{+}^{2}$.

As a further investigation of Theorem A, Fu et al. [7] gave the necessary and sufficient condition for the Schur-convexity of the generalized Heronian means $H_{p, w}(x, y)$, which is stated in the following theorem.

Theorem B. For fixed $(p, w) \in \mathbb{R}^{2}$, the generalized Heronian means $H_{p, w}(x, y)$ is Schur-convex for $(x, y) \in \mathbb{R}_{++}^{2}$ if and only if

$$
\begin{gathered}
(p, w) \in\{(p, w) \mid p \geq 2,0 \leq w \leq 2(p-1)\} \\
\cup\{(p, w) \mid 1<p \leq 2, w=0\} .
\end{gathered}
$$

Furthermore, $H_{p, w}(x, y)$ is Schur-concave for $(x, y) \in \mathbb{R}_{++}^{2}$ if and only if

$$
(p, w) \in\{(p, w) \mid p \leq 2, \max \{0,2(p-1)\} \leq w\} .
$$

Remark 1. It is easy to observe that, for $p=1, w=0$, $H_{1,0}(x, y)=(x+y) / 2$ is Schur-convex and Schur-concave for $(x, y) \in \mathbb{R}_{++}^{2}$. In addition, we note that $\{(p, w) \mid p=2, w=$ $0\} \subset\{(p, w) \mid p \geq 2,0 \leq w \leq 2(p-1)\}$. Thus, the conditions of Schur-convexity of $H_{p, w}(x, y)$ in Theorem B can be rewritten as

$$
\begin{gathered}
(p, w) \in\{(p, w) \mid p \geq 2,0 \leq w \leq 2(p-1)\} \\
\cup\{(p, w) \mid 1 \leq p<2, w=0\} .
\end{gathered}
$$

The Schur-power-convexity of $H_{p, w}(x, y)$ was investigated by Yang [8].

In 2006, Trif [9] considered the following generalized Muirhead means, defined by

$$
M(p, q ; x, y)=\left(\frac{x^{p} y^{q}+x^{q} y^{p}}{2}\right)^{1 /(p+q)},
$$

where $x, y \in \mathbb{R}_{++}, p, q \in \mathbb{R}, p+q \neq 0$.

Gong et al. [10] investigated the Schur-convexity of generalized Muirhead means $M(p, q ; x, y)$ and obtained the following results.

Theorem C. For fixed $(p, q) \in \mathbb{R}^{2}$, the generalized Muirhead means $M(p, q ; x, y)$ is Schur-convex for $(x, y) \in \mathbb{R}_{++}^{2}$ if and only if

$$
(p, q) \in\left\{(p, q) \mid(p-q)^{2} \geq p+q>0, p q \leq 0\right\} .
$$

Furthermore, $M(p, q ; x, y)$ is Schur-concave for $(x, y) \in$ $\mathbb{R}_{++}^{2}$ if and only if

$$
\begin{array}{r}
(p, q) \in\left\{(p, q) \mid p \geq 0, q \geq 0,(p-q)^{2} \leq p+q,\right. \\
(p, q) \neq(0,0)\} \cup\{(p, q) \mid p+q<0\} .
\end{array}
$$

Remark 2. If we define, for $p=0, q=0$, the generalized Muirhead means by $M(0,0 ; x, y)=\sqrt{x y}$, we can easily find that $M(0,0 ; x, y)$ is Schur-concave for $(x, y) \in \mathbb{R}_{++}^{2}$; thereby, the conditions of Schur-concave of $M(p, q ; x, y)$ in Theorem $\mathrm{C}$ can be rewritten as

$$
\begin{aligned}
(p, q) \in & \left\{(p, q) \mid p \geq 0, q \geq 0,(p-q)^{2} \leq p+q\right\} \\
& \cup\{(p, q) \mid p+q<0\} .
\end{aligned}
$$

The Schur-geometric-convexity and Schur-harmonicconvexity of the generalized Muirhead means $M(p, q ; x, y)$ were studied by Xia and Chu in $[11,12]$.

In this paper we generalize the generalized Muirhead means $M(p, q ; x, y)$ and the generalized Heronian means $H_{p, w}(x, y)$ in a unified form. For this purpose we define a generalized Muirhead-Heronian means $\mathscr{H}_{p, q, w}(x, y)$, as follows:

$$
\begin{aligned}
& \mathscr{H}_{p, q, w}(x, y) \\
& = \begin{cases}\left(\frac{x^{p} y^{q}+w(x y)^{(p+q) / 2}+x^{q} y^{p}}{2+w}\right)^{1 /(p+q)}, & p+q \neq 0, \\
\sqrt{x y}, & p=q=0,\end{cases}
\end{aligned}
$$

where $(p, q) \in \mathbb{R}^{2},(x, y) \in \mathbb{R}_{++}^{2}$.

The paper is organized as follows. Section 2 introduces several definitions and lemmas; Section 3 discusses the Schur-convexity of the generalized Muirhead-Heronian means; Section 4 provides some remarks on the results given in Theorem 9 and it is shown that the sufficient conditions of Schur-convexity of the generalized Heronian means $H_{p, w}(x, y)$ and the generalized Muirhead means $M(p, q ; x, y)$ can be deduced from Theorem 9 as special cases.

\section{Definitions and Lemmas}

We introduce and establish several definitions and lemmas, which will be used in the proofs of the main results in Section 3.

Definition 3 (see [13, page 7]). For any $x=\left(x_{1}, x_{2}, \ldots, x_{n}\right)$, $y=\left(y_{1}, y_{2}, \ldots, y_{n}\right) \in \mathbb{R}^{n}$, let $x_{[1]} \geq x_{[2]} \geq \cdots \geq x_{[n]}$ and $y_{[1]} \geq y_{[2]} \geq \cdots \geq y_{[n]}$ denote the components of $x$ and $y$ in decreasing order, respectively.

The $n$-tuple $y$ is said to majorize $x$ (or $x$ is to be majorized by $y$ ), in symbols $x \prec y$, if

$$
\sum_{i=1}^{k} x_{[i]} \leq \sum_{i=1}^{k} y_{[i]}
$$

holds for $k=1,2, \ldots, n-1$,

$$
\sum_{i=1}^{n} x_{i}=\sum_{i=1}^{n} y_{i}
$$


Definition 4 (see [13, page 54]). For any $x=\left(x_{1}, x_{2}, \ldots, x_{n}\right)$, $y=\left(y_{1}, y_{2}, \ldots, y_{n}\right) \in \Omega\left(\Omega \subset \mathbb{R}^{n}\right), \phi: \Omega \rightarrow \mathbb{R}$ is said to be a Schur-convex function on $\Omega$ if $x \prec y$ on $\Omega$ implies $\phi(x) \leq$ $\phi(y)$ and $\phi$ is said to be a Schur-concave function on $\Omega$ if and only if $-\phi$ is a Schur-convex function.

Lemma 5 (see [13, page 57]). Let $\Omega\left(\subset \mathbb{R}^{n}\right)$ be a symmetric convex set with nonempty interior $\Omega^{\circ}$ and $\phi: \Omega \rightarrow R$ a continuous symmetric function on $\Omega$. If $\phi$ is differentiable on $\Omega^{\circ}$, then $\phi$ is the Schur-convex (Schur-concave) function on $\Omega$ if and only if

$$
\left(x_{1}-x_{2}\right)\left(\frac{\partial \phi}{\partial x_{1}}-\frac{\partial \phi}{\partial x_{2}}\right) \geq 0 \quad(\leq 0)
$$

holds for all $\left(x_{1}, x_{2}, \ldots, x_{n}\right) \in \Omega^{o}$.

Lemma 6. Suppose that $p, q \in \mathbb{R}, p>q, \lambda \geq 1$ and

$$
\begin{aligned}
g(\lambda)= & p \lambda^{p-q}+q+\frac{w(p+q)}{2} \lambda^{(p-q) / 2} \\
& -q \lambda^{p-q+1}-p \lambda-\frac{w(p+q)}{2} \lambda^{((p-q) / 2)+1} .
\end{aligned}
$$

Suppose also that

$$
\begin{gathered}
B_{1}=\{(p, q, w) \mid p+q>0, p-q-2 \geq 0, w \geq 0\}, \\
B_{2}=\{(p, q, w) \mid p+q>0, p-q-2<0, w \geq 0\}, \\
E_{11}=B_{1} \\
\cap\{(p, q, w) \mid q<0, \\
\left.\quad 2(p-q)^{2}-(2+w)(p+q) \geq 0\right\}, \\
E_{12}=B_{2} \\
\cap\{(p, q, w) \mid q<0, \\
2(p-q)^{2}-(2+w)(p+q) \geq 0, \\
\quad(p+q)(p-q-2) w \\
-8 q(p-q+1) \geq 0\} .
\end{gathered}
$$

Then $(p+q) g(\lambda) \geq 0$ for $(p, q, w) \in E_{11} \cup E_{12}$.

Proof. Differentiating $g(\lambda)$ with respect to $\lambda$ gives

$$
\begin{aligned}
g^{\prime}(\lambda)= & p(p-q) \lambda^{p-q-1} \\
& +\frac{w(p+q)(p-q)}{4} \lambda^{((p-q) / 2)-1} \\
& -q(p-q+1) \lambda^{p-q}-p \\
& -\frac{w(p+q)(p-q+2)}{4} \lambda^{(p-q) / 2} \\
g^{\prime}(1) & =\frac{2(p-q)^{2}-(2+w)(p+q)}{2} .
\end{aligned}
$$

Let $f(\lambda)=\lambda^{q-p+2} g^{\prime \prime}(\lambda)$, then

$$
\begin{aligned}
f(\lambda)= & p(p-q)(p-q-1) \\
& +\frac{w(p+q)(p-q)(p-q-2)}{8} \lambda^{(q-p) / 2} \\
& -q(p-q+1)(p-q) \lambda \\
& -\frac{w(p+q)(p-q+2)(p-q)}{8} \lambda^{((q-p) / 2)+1}, \\
f(1) & =\frac{p-q}{2}\left(2(p-q)^{2}-(2+w)(p+q)\right) .
\end{aligned}
$$

Differentiating $f(\lambda)$ with respect to $\lambda$ yields

$$
\begin{aligned}
f^{\prime}(\lambda)= & -\frac{w(p+q)(p-q)^{2}(p-q-2)}{16} \lambda^{((q-p) / 2)-1} \\
& -q(p-q+1)(p-q) \\
& +\frac{w(p+q)(p-q+2)(p-q)(p-q-2)}{16} \lambda^{(q-p) / 2}, \\
f^{\prime}(1)= & \frac{p-q}{8}((p+q)(p-q-2) w-8 q(p-q+1)), \\
f^{\prime \prime}(\lambda) & \\
= & -\frac{w(p+q)(p-q)^{2}(p-q-2)(p-q+2)}{32} \\
& \times(\lambda-1) \lambda^{((q-p) / 2)-2} .
\end{aligned}
$$

In order to prove Lemma 6 , we need to consider the two cases below.

Case $1\left((p, q, w) \in E_{11}\right)$. In view of $(p, q, w) \in E_{11}$, we have $f^{\prime \prime}(\lambda) \leq 0$ for $\lambda \geq 1$. Hence $f^{\prime}(\lambda)$ is decreasing on $[1,+\infty)$, by which, together with

$$
\begin{gathered}
f^{\prime}(1)>0, \\
\lim _{\lambda \rightarrow+\infty} f^{\prime}(\lambda)=-q(p-q+1)(p-q)>0,
\end{gathered}
$$

we deduce that $f^{\prime}(\lambda)>0$ for $\lambda \geq 1$. This means that $f(\lambda)$ is increasing on $[1,+\infty)$. Thus, we have, for $\lambda \geq 1$,

$$
\begin{aligned}
f(\lambda) & \geq f(1) \geq 0 \\
& \Longrightarrow g^{\prime \prime}(\lambda)=\frac{f(\lambda)}{\lambda^{q-p+2}} \geq 0 \\
& \Longrightarrow g^{\prime}(\lambda) \geq g^{\prime}(1) \geq 0 \\
& \Longrightarrow g(\lambda) \geq g(1)=0 .
\end{aligned}
$$


Case $2\left((p, q, w) \in E_{12}\right)$. By $(p, q, w) \in E_{12}$, we have $f^{\prime \prime}(\lambda) \geq$ 0 for $\lambda \geq 1$. Thus $f^{\prime}(\lambda)$ is increasing on $[1,+\infty)$, by which, together with

$$
\begin{gathered}
f^{\prime}(1)>0, \\
\lim _{\lambda \rightarrow+\infty} f^{\prime}(\lambda)=-q(p-q+1)(p-q)>0,
\end{gathered}
$$

we obtain $f^{\prime}(\lambda)>0$ for $\lambda \geq 1$. It follows that $f(\lambda)$ is increasing on $[1,+\infty)$. Thus, we have, for $\lambda \geq 1$,

$$
\begin{aligned}
f(\lambda) & \geq f(1) \geq 0 \\
& \Longrightarrow g^{\prime \prime}(\lambda)=\frac{f(\lambda)}{\lambda^{q-p+2}} \geq 0 \\
& \Longrightarrow g^{\prime}(\lambda) \geq g^{\prime}(1) \geq 0 \\
& \Longrightarrow g(\lambda) \geq g(1)=0 .
\end{aligned}
$$

This implies that $(p+q) g(\lambda) \geq 0$. The proof of Lemma 6 is complete.

Lemma 7. Suppose that $p, q \in \mathbb{R}, p>q, \lambda \geq 1$ and

$$
\begin{aligned}
g(\lambda)= & p \lambda^{p-q}+q+\frac{w(p+q)}{2} \lambda^{(p-q) / 2} \\
& -q \lambda^{p-q+1}-p \lambda-\frac{w(p+q)}{2} \lambda^{((p-q) / 2)+1} .
\end{aligned}
$$

\section{Suppose also that}

$$
\begin{gathered}
B_{1}=\{(p, q, w) \mid p+q>0, p-q-2 \geq 0, w \geq 0\}, \\
B_{2}=\{(p, q, w) \mid p+q>0, p-q-2<0, w \geq 0\}, \\
B_{3}=\{(p, q, w) \mid p+q<0, p-q-2 \geq 0, w \geq 0\}, \\
B_{4}=\{(p, q, w) \mid p+q<0, p-q-2<0, w \geq 0\}, \\
E_{21}=B_{1} \cap\{(p, q, w) \mid q>0, \\
2(p-q)^{2}-(2+w)(p+q) \leq 0, \\
(p+q)(p-q-2) w \\
-8 q(p-q+1) \leq 0\}, \\
E_{22}=B_{2} \cap\{(p, q, w) \mid q>0, \\
\left.2(p-q)^{2}-(2+w)(p+q) \leq 0\right\}, \\
E_{23}=B_{3} \cap\{(p, q, w) \mid q<0, \\
2(p-q)^{2}-(2+w)(p+q)>0, \\
(p+q)(p-q-2) w \\
-8 q(p-q+1) \geq 0\}, \\
E_{24}=B_{4} \cap\{(p, q, w) \mid q<0, \\
\left.2(p-q)^{2}-(2+w)(p+q)>0\right\} .
\end{gathered}
$$

Case 1. If $(p, q, w) \in E_{21}$, then

$$
f^{\prime}(1) \leq 0, \quad f(1) \leq 0, \quad g^{\prime}(1) \leq 0, \quad f^{\prime \prime}(\lambda) \leq 0 .
$$

Thus we have, for $\lambda \in[1,+\infty)$,

$$
\begin{aligned}
f^{\prime \prime}(\lambda) & \leq 0 \\
& \Longrightarrow f^{\prime}(\lambda) \text { is decreasing } \\
& \Longrightarrow f^{\prime}(\lambda) \leq 0 \\
& \Longrightarrow f(\lambda) \text { is decreasing } \Longrightarrow f(\lambda) \leq 0 \\
& \Longrightarrow g^{\prime \prime}(\lambda) \leq 0 \\
& \Longrightarrow g^{\prime}(\lambda) \text { is decreasing } \Longrightarrow g^{\prime}(\lambda) \leq 0 \\
& \Longrightarrow g(\lambda) \text { is decreasing } \Longrightarrow g(\lambda) \leq g(1)=0 \\
& \Longrightarrow(p+q) g(\lambda) \leq 0 .
\end{aligned}
$$

Then $(p+q) g(\lambda) \leq 0$ for $(p, q, w) \in E_{21} \cup E_{22} \cup E_{23} \cup E_{24}$. 
Case 2. If $(p, q, w) \in E_{22}$, then

$$
\begin{gathered}
f^{\prime}(1)<0 \\
\lim _{\lambda \rightarrow+\infty} f^{\prime}(\lambda)=-q(p-q+1)(p-q)<0, \\
f(1) \leq 0, \quad g^{\prime}(1) \leq 0, \\
f^{\prime \prime}(\lambda) \geq 0 .
\end{gathered}
$$

Thus we have, for $\lambda \in[1,+\infty)$,

$$
\begin{aligned}
f^{\prime \prime}(\lambda) & \geq 0 \\
& \Longrightarrow f^{\prime}(\lambda) \text { is increasing } \\
& \Longrightarrow f^{\prime}(\lambda) \leq 0 \\
& \Longrightarrow f(\lambda) \text { is decreasing } \Longrightarrow f(\lambda) \leq 0 \\
& \Longrightarrow g^{\prime \prime}(\lambda) \leq 0 \\
& \Longrightarrow g^{\prime}(\lambda) \text { is decreasing } \Longrightarrow g^{\prime}(\lambda) \leq 0 \\
& \Longrightarrow g(\lambda) \text { is decreasing } \Longrightarrow g(\lambda) \leq g(1)=0 \\
& \Longrightarrow(p+q) g(\lambda) \leq 0 .
\end{aligned}
$$

Case 3. If $(p, q, w) \in E_{23}$, then

$$
f^{\prime}(1) \geq 0, \quad f(1)>0, \quad g^{\prime}(1)>0, \quad f^{\prime \prime}(\lambda) \geq 0 \text {. }
$$

Thus we have, for $\lambda \in[1,+\infty)$,

$$
\begin{aligned}
f^{\prime \prime}(\lambda) & \geq 0 \\
& \Longrightarrow f^{\prime}(\lambda) \text { is increasing } \\
& \Longrightarrow f^{\prime}(\lambda) \geq 0 \Longrightarrow f(\lambda) \text { is increasing } \\
& \Longrightarrow f(\lambda)>0 \Longrightarrow g^{\prime \prime}(\lambda)>0 \\
& \Longrightarrow g^{\prime}(\lambda) \text { is increasing } \Longrightarrow g^{\prime}(\lambda)>0 \\
& \Longrightarrow g(\lambda) \text { is increasing } \Longrightarrow g(\lambda) \geq g(1)=0 \\
& \Longrightarrow(p+q) g(\lambda) \leq 0 .
\end{aligned}
$$

Case 4. If $(p, q, w) \in E_{24}$, then

$$
\begin{gathered}
f^{\prime}(1)>0, \quad f(1)>0, \\
\lim _{\lambda \rightarrow+\infty} f^{\prime}(\lambda)=-q(p-q+1)(p-q)>0, \\
g^{\prime}(1)>0, \quad f^{\prime \prime}(\lambda)<0 .
\end{gathered}
$$

Thus we have, for $\lambda \in[1,+\infty)$,

$$
\begin{aligned}
f^{\prime \prime}(\lambda) & <0 \\
& \Longrightarrow f^{\prime}(\lambda) \text { is decreasing } \Longrightarrow f^{\prime}(\lambda)>0 \\
& \Longrightarrow f(\lambda) \text { is increasing } \Longrightarrow f(\lambda)>0 \\
& \Longrightarrow g^{\prime \prime}(\lambda)>0 \Longrightarrow g^{\prime}(\lambda) \text { is increasing } \\
& \Longrightarrow g^{\prime}(\lambda)>0 \Longrightarrow g(\lambda) \text { is increasing } \\
& \Longrightarrow g(\lambda) \geq g(1)=0 \Longrightarrow(p+q) g(\lambda) \leq 0 .
\end{aligned}
$$

This completes the proof of Lemma 7.

Lemma 8. Suppose that $p, q \in \mathbb{R}, p>q, \lambda \geq 1$ and

$$
\begin{aligned}
g(\lambda)= & p \lambda^{p-q}+q+\frac{w(p+q)}{2} \lambda^{(p-q) / 2} \\
& -q \lambda^{p-q+1}-p \lambda-\frac{w(p+q)}{2} \lambda^{((p-q) / 2)+1} .
\end{aligned}
$$

Suppose also that

$$
\begin{gathered}
B_{1}=\{(p, q, w) \mid p+q>0, p-q-2 \geq 0, w \geq 0\}, \\
B_{3}=\{(p, q, w) \mid p+q<0, p-q-2 \geq 0, w \geq 0\}, \\
E_{31}=B_{1} \cap\{(p, q, w) \mid q>0, \\
(p+q)(p-q-2) w-8 q(p-q+1)>0, \\
\left.2(p-q)^{2}-(2+w)(p+q)<0\right\} \\
\cap\left\{(p, q, w) \mid(p-q)^{2}-3(p+q)+2 \leq 0\right\}, \\
E_{32}=B_{3} \cap\{(p, q, w) \mid q<0,(p+q)(p-q-2) w \\
-8 q(p-q+1)<0\} .
\end{gathered}
$$$$
\text { Then }(p+q) g(\lambda) \leq 0 \text { for }(p, q, w) \in E_{31} \cup E_{32} \text {. }
$$

Proof. Based on the differential expressions $g^{\prime}(\lambda), g^{\prime \prime}(\lambda)$, $g^{\prime}(1), f^{\prime}(\lambda), f^{\prime \prime}(\lambda), f^{\prime}(1)$ obtained in the proof of Lemma 6 , in order to prove Lemma 8 , we need to consider the two cases below.

Case 1. If $(p, q, w) \in E_{31}$, then

$$
\begin{gathered}
f^{\prime \prime}(\lambda) \leq 0, \quad f^{\prime}(1)>0, \\
\lim _{\lambda \rightarrow+\infty} f^{\prime}(\lambda)=-q(p-q+1)(p-q)<0 .
\end{gathered}
$$

Hence, we deduce that there exists $\lambda_{1} \in(1,+\infty)$ such that $f^{\prime}\left(\lambda_{1}\right)=0$, satisfying $f^{\prime}(\lambda)>0$ for $\lambda \in\left[1, \lambda_{1}\right)$ and $f^{\prime}(\lambda)<0$ for $\lambda \in\left(\lambda_{1},+\infty\right)$. 
Further, we conclude that $f(\lambda)$ is increasing on $\left[1, \lambda_{1}\right)$ and decreasing on $\left(\lambda_{1},+\infty\right)$; thereby, we get $f(\lambda) \leq f\left(\lambda_{1}\right)$ for $\lambda \epsilon$ $[1,+\infty)$.

From $f^{\prime}\left(\lambda_{1}\right)=0$ we have

$$
\begin{aligned}
& \left(\frac{w(p+q)(p-q+2)(p-q)(p-q-2)}{16}\right. \\
& \left.-\frac{w(p+q)(p-q)^{2}(p-q-2)}{16 \lambda_{1}}\right) \lambda_{1}^{(q-p) / 2} \\
& =q(p-q+1)(p-q) ;
\end{aligned}
$$

this yields

$$
\begin{aligned}
& \lambda_{1}^{(q-p) / 2} \\
& \quad=\frac{16 q(p-q+1) \lambda_{1}}{(p+q)\left((p-q+2) \lambda_{1}+q-p\right)(p-q-2) w} ;
\end{aligned}
$$

we thus have

$$
\begin{aligned}
f\left(\lambda_{1}\right)= & p(p-q)(p-q-1) \\
& -q(p-q+1)(p-q) \lambda_{1} \\
& +\left(\frac{w(p+q)(p-q)(p-q-2)}{8}\right. \\
& \left.\quad-\frac{w(p+q)(p-q+2)(p-q) \lambda_{1}}{8}\right) \lambda_{1}^{(q-p) / 2} \\
= & \frac{(p-q)^{2} G_{1}\left(\lambda_{1}\right)}{(p-q-2)\left((p-q+2) \lambda_{1}+q-p\right)},
\end{aligned}
$$

where

$$
\begin{aligned}
G_{1}\left(\lambda_{1}\right)= & -q(p-q+2)(p-q+1)\left(\lambda_{1}-1\right)^{2} \\
& +\left((p-q)^{2}-3(p+q)+2\right) \\
& \times\left(2+(p-q+2)\left(\lambda_{1}-1\right)\right)<0 .
\end{aligned}
$$

Note that $(p, q, w) \in E_{31}$ implies $p-q-2>0$ and

$$
\begin{aligned}
& (p-q+2) \lambda_{1}+q-p \\
& \quad>(p-q+2)+q-p=2 ;
\end{aligned}
$$

we conclude that $f(\lambda) \leq f\left(\lambda_{1}\right)<0$ for $\lambda \in[1,+\infty)$.

Hence, from $g^{\prime}(1)<0$, one has, for $\lambda \in[1,+\infty)$,

$$
\begin{aligned}
f(\lambda) & <0 \\
& \Longrightarrow g^{\prime \prime}(\lambda)<0 \Longrightarrow g^{\prime}(\lambda) \text { is decreasing } \\
& \Longrightarrow g^{\prime}(\lambda)<0 \Longrightarrow g(\lambda) \text { is decreasing } \\
& \Longrightarrow g(\lambda) \leq g(1)=0 \Longrightarrow(p+q) g(\lambda) \leq 0 .
\end{aligned}
$$

Case 2. If $(p, q, w) \in E_{32}$, then

$$
\begin{gathered}
f^{\prime \prime}(\lambda) \geq 0, \quad f^{\prime}(1)<0, \\
\lim _{\lambda \rightarrow+\infty} f^{\prime}(\lambda)=-q(p-q+1)(p-q)>0 .
\end{gathered}
$$

Thus, we deduce that there exists $\lambda_{2} \in(1,+\infty)$ such that $f^{\prime}\left(\lambda_{2}\right)=0$, satisfying $f^{\prime}(\lambda)<0$ for $\lambda \in\left[1, \lambda_{2}\right)$ and $f^{\prime}(\lambda)>0$ for $\lambda \in\left(\lambda_{2},+\infty\right)$.

It follows that $f(\lambda)$ is decreasing on $\left[1, \lambda_{2}\right)$ and increasing on $\left(\lambda_{2},+\infty\right)$, therefore, we obtain

$$
f(\lambda) \geq f\left(\lambda_{2}\right) \quad \text { for } \lambda \in[1,+\infty) .
$$

From $f^{\prime}\left(\lambda_{2}\right)=0$, we have

$$
\begin{aligned}
& \left(\frac{w(p+q)(p-q+2)(p-q)(p-q-2)}{16}\right. \\
& \left.-\frac{w(p+q)(p-q)^{2}(p-q-2)}{16 \lambda_{2}}\right) \lambda_{2}^{(q-p) / 2} \\
& \quad=q(p-q+1)(p-q) ;
\end{aligned}
$$

that is,

$$
\begin{aligned}
& \lambda_{2}^{(q-p) / 2} \\
& \quad=\frac{16 q(p-q+1) \lambda_{2}}{(p+q)\left((p-q+2) \lambda_{2}+q-p\right)(p-q-2) w} ;
\end{aligned}
$$

we thus have

$$
\begin{aligned}
f\left(\lambda_{2}\right)= & p(p-q)(p-q-1) \\
& -q(p-q+1)(p-q) \lambda_{2} \\
& +\left(\frac{w(p+q)(p-q)(p-q-2)}{8}\right. \\
& \left.\quad-\frac{w(p+q)(p-q+2)(p-q) \lambda_{2}}{8}\right) \lambda_{2}^{(q-p) / 2} \\
= & \frac{(p-q)^{2} G_{2}\left(\lambda_{2}\right)}{(p-q-2)\left((p-q+2) \lambda_{2}+q-p\right)},
\end{aligned}
$$

where

$$
\begin{aligned}
G_{2}\left(\lambda_{2}\right)= & -q(p-q+2)(p-q+1)\left(\lambda_{2}-1\right)^{2} \\
& +\left((p-q)^{2}-3(p+q)+2\right) \\
& \times\left(2+(p-q+2)\left(\lambda_{2}-1\right)\right)>0 .
\end{aligned}
$$

Note that $(p, q, w) \in E_{32}$ implies $p-q-2>0$ and

$$
\begin{aligned}
& (p-q+2) \lambda_{2}+q-p \\
& \quad>(p-q+2)+q-p=2,
\end{aligned}
$$

which yields that $f(\lambda) \geq f\left(\lambda_{2}\right)>0$ for $\lambda \in[1,+\infty)$. 
Therefore, from $g^{\prime}(1)>0$, one has, for $\lambda \in[1,+\infty)$, $f(\lambda)>0$

$$
\begin{aligned}
& \Longrightarrow g^{\prime \prime}(\lambda)>0 \Longrightarrow g^{\prime}(\lambda) \text { is increasing } \\
& \Longrightarrow g^{\prime}(\lambda)>0 \Longrightarrow g(\lambda) \text { is increasing } \\
& \Longrightarrow g(\lambda) \geq g(1)=0 \Longrightarrow(p+q) g(\lambda) \leq 0 .
\end{aligned}
$$

The proof of Lemma 8 is completed.

\section{Main Result}

The main result of this paper is given by Theorem 9 below.

Theorem 9. For fixed $(p, q, w) \in \mathbb{R}^{3}$, let

$$
\begin{aligned}
& A_{1}=\{(p, q, w) \mid p-q-2 \geq 0, q \leq 0\} \\
& \cup\{(p, q, w) \mid p-q-2<0, q \leq 0, \\
& (p+q)(p-q-2) w \\
& -8 q(p-q+1) \geq 0\}, \\
& A_{2}=\{(p, q, w) \mid q-p-2 \geq 0, p \leq 0\} \\
& \cup\{(p, q, w) \mid q-p-2<0, p \leq 0, \\
& (p+q)(q-p-2) w \\
& -8 p(q-p+1) \geq 0\}, \\
& A_{3}=\{(p, q, w) \mid p-q-2 \geq 0, q>0, \\
& (p+q)(p-q-2) w \\
& -8 q(p-q+1) \leq 0\} \\
& \cup\{(p, q, w) \mid p>q, p-q-2<0, q>0\}, \\
& A_{4}=\{(p, q, w) \mid q-p-2 \geq 0, p>0, \\
& (p+q)(q-p-2) w \\
& -8 p(q-p+1) \leq 0\} \\
& \cup\{(p, q, w) \mid q>p, q-p-2<0, p>0\}, \\
& A_{5}=\{(p, q, w) \mid p-q-2 \geq 0, q<0, \\
& (p+q)(p-q-2) w \\
& -8 q(p-q+1) \geq 0\} \\
& \cup\{(p, q, w) \mid p>q, p-q-2<0, q<0\}, \\
& A_{6}=\{(p, q, w) \mid q-p-2 \geq 0, p<0, \\
& (p+q)(q-p-2) w \\
& -8 p(q-p+1) \geq 0\} \\
& \cup\{(p, q, w) \mid q>p, q-p-2<0, p<0\},
\end{aligned}
$$

$$
\begin{aligned}
A_{7}=\{ & (p, q, w) \mid p-q-2 \geq 0, q>0, \\
& (p+q)(p-q-2) w \\
& -8 q(p-q+1)>0\}, \\
A_{8}=\{(p, q, w) \mid q-p-2 \geq 0, p>0, & \\
& (p+q)(q-p-2) w \\
& -8 p(q-p+1)>0\}, \\
A_{9}=\{ & (p, q, w) \mid p-q-2 \geq 0, q<0, \\
& (p+q)(p-q-2) w \\
& -8 q(p-q+1)<0\}, \\
A_{10}=\{ & (p, q, w) \mid q-p-2 \geq 0, p<0, \\
& (p+q)(q-p-2) w \\
& -8 p(q-p+1)<0\},
\end{aligned}
$$

and let

$$
\begin{aligned}
& S_{1}=\{((p, q, w) \mid p+q>0,2(p-q)^{2} \\
&-(2+w)(p+q) \geq 0, w \geq 0\} \\
& \cap\left(A_{1} \cup A_{2}\right), \\
& S_{2}=\{(p, q, w) \mid p=q, w \geq 0\} \\
& \cup\{(p, q, w) \mid p \leq 2, q=0, \max \{0,2(p-1)\} \leq w\} \\
& \cup\{(p, q, w) \mid q \leq 2, p=0, \max \{0,2(q-1)\} \leq w\} \\
& \cup {[\{(p, q, w) \mid p+q>0, w \geq 0,} \\
&\left.\left.2(p-q)^{2}-(2+w)(p+q) \leq 0\right\} \cap\left(A_{3} \cup A_{4}\right)\right] \\
& \cup {[\{(p, q, w) \mid p+q<0, w \geq 0,} \\
&\left.\left.2(p-q)^{2}-(2+w)(p+q)>0\right\} \cap\left(A_{5} \cup A_{6}\right)\right] \\
& \cup {[\{(p, q, w) \mid p+q>0, w \geq 0,} \\
&(p-q)^{2}-3(p+q)+2 \leq 0, \\
&\left.2(p-q)^{2}-(2+w)(p+q)<0\right\} \\
&\left.\cap\left(A_{7} \cup A_{8}\right)\right] \\
& \cup {\left[\{(p, q, w) \mid p+q<0, w \geq 0\} \cap\left(A_{9} \cup A_{10}\right)\right] . }
\end{aligned}
$$

The following assertions holds true.

(1) If $(p, q, w) \in S_{1}$, then the generalized Muirhead-Heronian means $\mathscr{H}_{p, q, w}(x, y)$ is Schur-convex for $(x, y) \in$ $\mathbb{R}_{++}^{2}$. 
(2) If $(p, q, w) \in S_{2}$, then the generalized Muirhead-Heronian means $\mathscr{H}_{p, q, w}(x, y)$ is Schur-concave for $(x, y) \in$ $\mathbb{R}_{++}^{2}$.

Proof. Note that the expression $\mathscr{H}_{p, q, w}(x, y)$ is of symmetry between $x$ and $y$ and without loss of generality we assume that $x \geq y$.

Case 1. If $p=q$, then $\mathscr{H}_{p, q, w}(x, y)=\sqrt{x y}$. Define

$$
(x-y)\left(\frac{\partial \mathscr{H}}{\partial x}-\frac{\partial \mathscr{H}}{\partial y}\right)=\frac{-(x-y)^{2}}{2 \sqrt{x y}} \leq 0 .
$$

Hence, $\mathscr{H}_{p, q, w}(x, y)$ is Schur-concave for $(x, y) \in \mathbb{R}_{++}^{2}$.

Case 2. If $q=0$, we have the following known results (see Theorem B and Remark 1 in Section 1).

if

$\mathscr{H}_{p, 0, w}(x, y)$ is Schur-convex for $(x, y) \in \mathbb{R}_{++}^{2}$ if and only

$$
\begin{gathered}
(p, w) \in\{(p, w) \mid p \geq 2,0 \leq w \leq 2(p-1)\} \\
\cup\{(p, w) \mid 1 \leq p<2, w=0\} ;
\end{gathered}
$$

if

$$
\mathscr{H}_{p, 0, w}(x, y) \text { is Schur-concave for }(x, y) \in \mathbb{R}_{++}^{2} \text { if and only }
$$

$$
(p, w) \in\{(p, w) \mid p \leq 2, \max \{0,2(p-1)\} \leq w\} .
$$

Case 3. If $q \neq 0$, then

$$
\begin{aligned}
(x-y)\left(\frac{\partial \mathscr{H}}{\partial x}-\frac{\partial \mathscr{H}}{\partial y}\right) \\
=\frac{(x-y) \mathscr{H}_{p, q, w}(x, y) F(x, y)}{x^{p} y^{q}+w(x y)^{(p+q) / 2}+x^{q} y^{p}},
\end{aligned}
$$

where

$$
\begin{aligned}
& F(x, y) \\
& =\frac{y^{p+q-1}}{p+q}\left(p\left(\frac{x}{y}\right)^{p-1}+q\left(\frac{x}{y}\right)^{q-1}\right. \\
& +\frac{w(p+q)}{2}\left(\frac{x}{y}\right)^{((p+q) / 2)-1} \\
& -q\left(\frac{x}{y}\right)^{p}-p\left(\frac{x}{y}\right)^{q} \\
& \left.-\frac{w(p+q)}{2}\left(\frac{x}{y}\right)^{(p+q) / 2}\right)
\end{aligned}
$$

$$
\begin{aligned}
& =\frac{y^{p+q-1}}{p+q}\left(p \lambda^{p-1}+q \lambda^{q-1}\right. \\
& +\frac{w(p+q)}{2} \lambda^{((p+q) / 2)-1}-q \lambda^{p}-p \lambda^{q} \\
& \left.-\frac{w(p+q)}{2} \lambda^{(p+q) / 2}\right) \\
& =\frac{\lambda^{q-1} y^{p+q-1}}{p+q}\left(p \lambda^{p-q}+q\right. \\
& +\frac{w(p+q)}{2} \lambda^{(p-q) / 2}-q \lambda^{p-q+1} \\
& -\frac{\lambda^{q-1} y^{p+q-1}}{p+q} g(\lambda), \\
& \left.=\frac{w(p+q)}{2} \lambda^{((p-q) / 2)+1}\right)
\end{aligned}
$$

where $\lambda=x / y \geq 1$; in addition, the definition of $\mathscr{H}_{p, q, w}(x, y)$ implies that $p+q \neq 0$.

\section{Using Lemma 6 gives}

$$
(p+q) g(\lambda) \geq 0 \quad \text { for }(p, q, w) \in E_{11} \cup E_{12} \text {, }
$$

where

$$
\begin{gathered}
E_{11}=B_{1} \cap\{(p, q, w) \mid q<0, \\
\left.2(p-q)^{2}-(2+w)(p+q) \geq 0\right\}, \\
E_{12}=B_{2} \cap\{(p, q, w) \mid q<0, \\
2(p-q)^{2}-(2+w)(p+q) \geq 0, \\
(p+q)(p-q-2) w \\
-8 q(p-q+1) \geq 0\} \\
B_{1}=\{(p, q, w) \mid p+q>0, p-q-2 \geq 0, w \geq 0\}, \\
B_{2}=\{(p, q, w) \mid p+q>0, p-q-2<0, w \geq 0\} .
\end{gathered}
$$

On the other hand, we deduce from the symmetry of $\mathscr{H}_{p, q, w}(x, y)$ with respect to $p$ and $q$ that

$$
(p+q) g(\lambda) \geq 0 \quad \text { for }(p, q, w) \in E_{11}^{\prime} \cup E_{12}^{\prime}
$$

where

$$
\begin{aligned}
& E_{11}^{\prime} \\
& =B_{1}^{\prime} \cap\{(p, q, w) \mid p<0, \\
& \left.\quad 2(p-q)^{2}-(2+w)(p+q) \geq 0\right\},
\end{aligned}
$$




$$
\begin{aligned}
& E_{12}^{\prime}, \\
& =B_{2}^{\prime} \cap\{(p, q, w) \mid p<0, \\
& \quad 2(p-q)^{2}-(2+w)(p+q) \geq 0, \\
& \quad(p+q)(q-p-2) w-8 p(q-p+1) \geq 0\}, \\
& B_{1}^{\prime}=\{(p, q, w) \mid p+q>0, \\
& \quad q-p-2 \geq 0, w \geq 0\}, \\
& B_{2}^{\prime}=\{(p, q, w) \mid p+q>0, \\
& q-p-2<0, w \geq 0\} .
\end{aligned}
$$

Now, by using Lemma 5 and combining the result stated in Case 2 , we deduce that $\mathscr{H}_{p, q, w}(x, y)$ is Schur-convex under the conditions below:

$$
\begin{aligned}
(p, q, w) & \\
\in & E_{11} \cup E_{12} \cup E_{11}^{\prime} \cup E_{12}^{\prime} \\
& \cup\{(p, q, w) \mid q=0, p \geq 2,0 \leq w \leq 2(p-1)\} \\
& \cup\{(p, q, w) \mid q=0,1 \leq p<2, w=0\} \\
& \cup\{(p, q, w) \mid p=0, q \geq 2,0 \leq w \leq 2(q-1)\} \\
& \cup\{(p, q, w) \mid p=0,1 \leq q<2, w=0\} \\
= & \{(p, q, w) \mid p+q>0, \\
& \left.2(p-q)^{2}-(2+w)(p+q) \geq 0, w \geq 0\right\} \\
& \cap\left(A_{1} \cup A_{2}\right)=S_{1} .
\end{aligned}
$$

This proves the validity of the first assertion in Theorem 9.

It is easy to find that

$$
\begin{aligned}
& E_{21} \cup E_{22} \\
& =A_{3} \cap\{(p, q, w) \mid p+q>0, w \geq 0, \\
& \left.\quad 2(p-q)^{2}-(2+w)(p+q) \leq 0\right\} .
\end{aligned}
$$

In view of the symmetry of $\mathscr{H}_{p, q, w}(x, y)$ between $p$ and $q$, utilizing a positional exchange between $p$ and $q$ in the above expression gives

$$
\begin{aligned}
& E_{21}^{\prime} \cup E_{22}^{\prime} \\
& =A_{4} \cap\{(p, q, w) \mid p+q>0, w \geq 0, \\
& \left.\quad 2(p-q)^{2}-(2+w)(p+q) \leq 0\right\} .
\end{aligned}
$$

Hence, we deduce from Lemma 7 that

$$
(p+q) g(\lambda) \leq 0 \quad \text { for } E_{21} \cup E_{22} \cup E_{21}^{\prime} \cup E_{22}^{\prime}
$$

where

$$
\begin{aligned}
E_{21} \cup & E_{22} \cup E_{21}^{\prime} \cup E_{22}^{\prime} \\
= & \{(p, q, w) \mid p+q>0, w \geq 0, \\
& \left.2(p-q)^{2}-(2+w)(p+q) \leq 0\right\} \\
& \cap\left(A_{3} \cup A_{4}\right) .
\end{aligned}
$$

By using the same method as above, we can deduce that

$$
\begin{array}{r}
(p+q) g(\lambda) \leq 0 \\
\text { for }\left(E_{23} \cup E_{24} \cup E_{23}^{\prime} \cup E_{24}^{\prime}\right) \\
\cup\left(E_{31} \cup E_{31}^{\prime}\right) \cup\left(E_{32} \cup E_{32}^{\prime}\right),
\end{array}
$$

where

$$
\begin{aligned}
E_{23} \cup & E_{24} \cup E_{23}^{\prime} \cup E_{24}^{\prime} \\
= & \{(p, q, w) \mid p+q<0, w \geq 0, \\
& \left.2(p-q)^{2}-(2+w)(p+q)>0\right\} \\
& \cap\left(A_{5} \cup A_{6}\right), \\
E_{31} \cup & E_{31}^{\prime} \\
= & \{(p, q, w) \mid p+q>0, w \geq 0, \\
& (p-q)^{2}-3(p+q)+2 \leq 0, \\
& \left.2(p-q)^{2}-(2+w)(p+q)<0\right\} \\
& \cap\left(A_{7} \cup A_{8}\right), \\
E_{32} \cup & E_{32}^{\prime} \\
= & \{(p, q, w) \mid p+q<0, w \geq 0\} \\
\cap & \left(A_{9} \cup A_{10}\right) .
\end{aligned}
$$

Therefore, we deduce from Lemma 5 and the results stated in Cases 1 and 2 that $\mathscr{H}_{p, q, w}(x, y)$ is Schur-concave under the following conditions:

$(p, q, w)$

$$
\begin{aligned}
\in & \left(E_{21} \cup E_{22} \cup E_{21}^{\prime} \cup E_{22}^{\prime}\right) \\
& \cup\left(E_{23} \cup E_{24} \cup E_{23}^{\prime} \cup E_{24}^{\prime}\right) \\
& \cup\left(E_{31} \cup E_{31}^{\prime}\right) \cup\left(E_{32} \cup E_{32}^{\prime}\right) \\
& \cup\{(p, q, w) \mid p=q, w \geq 0\} \\
& \cup\{(p, q, w) \mid p \leq 2, q=0, \max \{0,2(p-1)\} \leq w\} \\
& \cup\{(p, q, w) \mid q \leq 2, p=0, \max \{0,2(q-1)\} \leq w\} \\
= & S_{2} .
\end{aligned}
$$


This proves the validity of the second assertion in Theorem 9 . The proof of Theorem 9 is thus completed.

\section{Some Remarks on the Results of Theorem 9}

In this section, we provide some remarks on the results given in Theorem 9; we show that the sufficient conditions of Schurconvexity of the generalized Heronian means $H_{p, w}(x, y)$ and the generalized Muirhead means $M(p, q ; x, y)$ can be deduced from Theorem 9 as special cases.

Remark 10. If we take $q=0$ in Theorem 9, we have $\mathscr{H}_{p, q, w}(x, y)=H_{p, w}(x, y)$. Furthermore, we have

$$
\begin{aligned}
S_{1}= & \{(p, q, w) \mid p>0, q=0,0 \leq w \leq 2(p-1)\} \\
\cap & {[\{(p, q, w) \mid p \geq 2, q=0, w \geq 0\}} \\
& \cup\{(p, q, w) \mid p<2, q=0, w=0\}] \\
= & \{(p, q, w) \mid p \geq 2, q=0,0 \leq w \leq 2(p-1)\} \\
& \cup\{(p, q, w) \mid 1 \leq p<2, q=0, w=0\},
\end{aligned}
$$

which are the sufficient conditions of Schur-convex of the generalized Heronian means $H_{p, w}(x, y)$ asserted by Theorem B.

On the other hand, we note that, for $q=0$,

$$
\begin{aligned}
& \{(p, q, w) \mid p+q>0, w \geq 0, \\
& \left.2(p-q)^{2}-(2+w)(p+q) \leq 0\right\} \\
& \cap\left(A_{3} \cup A_{4}\right)=\emptyset, \\
& \{(p, q, w) \mid p+q<0, w \geq 0 \\
& \left.2(p-q)^{2}-(2+w)(p+q)>0\right\} \\
& \cap\left(A_{5} \cup A_{6}\right) \\
& =\{(p, q, w) \mid p<0, q=0, w \geq 0\} \\
& \cap[\{(p, q, w) \mid p \leq-2, q=0, \\
& (-p-2) w \leq 8(-p+1)\} \\
& \cup\{(p, q, w) \mid-2<p<0, q=0, w \geq 0\}], \\
& \left\{(p, q, w) \mid p+q>0,(p-q)^{2}-3(p+q)+2 \leq 0,\right. \\
& \left.2(p-q)^{2}-(2+w)(p+q)<0\right\} \\
& \cap\left(A_{7} \cup A_{8}\right)=\emptyset,
\end{aligned}
$$

$$
\begin{aligned}
& \{(p, q, w) \mid p+q<0, w \geq 0\} \\
& \cap\left(A_{9} \cup A_{10}\right) \\
& =\{(p, q, w) \mid p<0, q=0, w \geq 0\} \\
& \cap\{(p, q, w) \mid p \leq-2, q=0, \\
& (-p-2) w>8(-p+1)\} .
\end{aligned}
$$

Hence, the set $S_{2}$ given in Theorem 9 reduces to the following form:

$$
\begin{aligned}
S_{2}= & \{(p, q, w) \mid p=0, q=0, w \geq 0\} \\
& \cup\{(p, q, w) \mid p \leq 2, q=0, \max \{0,2(p-1)\} \leq w\} \\
& \cup\{(p, q, w) \mid q=0, p=0, w \geq 0\} \\
& \cup\{(p, q, w) \mid p<0, q=0, w \geq 0\} \\
= & \{(p, q, w) \mid p \leq 2, q=0, \max \{0,2(p-1)\} \leq w\} .
\end{aligned}
$$

These are the sufficient conditions of Schur-concave of the generalized Heronian means $H_{p, w}(x, y)$ stated by Theorem $\mathrm{B}$.

Remark 11. If we put $w=0$ in Theorem 9, we have $\mathscr{H}_{p, q, w}(x, y)=M(p, q ; x, y)$. Furthermore, we have

$$
\begin{gathered}
S_{1}=\left\{(p, q, w) \mid p+q>0,(p-q)^{2} \geq p+q, w=0\right\} \\
\cap(\{(p, q, w) \mid q \leq 0, w=0\} \\
\cup\{(p, q, w) \mid p \leq 0, w=0\}) \\
=\{(p, q, w) \mid p+q>0, p q \leq 0, \\
\left.(p-q)^{2} \geq p+q, w=0\right\},
\end{gathered}
$$

which are the sufficient conditions of Schur-convex of the generalized Muirhead means $M(p, q ; x, y)$ given by Theorem C.

In addition, for $w=0$, we have

$$
\begin{gathered}
\{(p, q, w) \mid p+q>0, w \geq 0, \\
\left.2(p-q)^{2}-(2+w)(p+q) \leq 0\right\} \\
\cap\left(A_{3} \cup A_{4}\right) \\
=\{(p, q, w) \mid p>0, q>0, p \neq q, \\
\left.(p-q)^{2} \leq p+q, w=0\right\},
\end{gathered}
$$




$$
\begin{gathered}
\{(p, q, w) \mid p+q<0, w \geq 0, \\
\left.2(p-q)^{2}-(2+w)(p+q)>0\right\} \\
\cap\left(A_{5} \cup A_{6}\right) \\
\quad=\{(p, q, w) \mid p+q<0, p \neq q, w=0\}, \\
\left\{(p, q, w) \mid p+q>0,(p-q)^{2}-3(p+q)+2 \leq 0,\right. \\
\left.2(p-q)^{2}-(2+w)(p+q)<0\right\} \\
\cap\left(A_{7} \cup A_{8}\right)=\emptyset, \\
\{(p, q, w) \mid p+q<0, w \geq 0\} \cap\left(A_{9} \cup A_{10}\right)=\emptyset .
\end{gathered}
$$

Thus, the set $S_{2}$ given in Theorem 9 reduces to the following form:

$$
\begin{aligned}
S_{2}= & \{(p, q, w) \mid p=q, w=0\} \\
& \cup\{(p, q, w) \mid p \leq 1, q=0, w=0\} \\
& \cup\{(p, q, w) \mid q \leq 1, p=0, w=0\} \\
& \cup\{(p, q, w) \mid p>0, q>0, p \neq q, \\
& \left.(p-q)^{2} \leq p+q, w=0\right\} \\
& \cup\{(p, q, w) \mid p+q<0, p \neq q, w=0\} \\
= & \left\{(p, q, w) \mid p \geq 0, q \geq 0,(p-q)^{2} \leq p+q, w=0\right\} \\
& \cup\{(p, q, w) \mid p+q<0, w=0\} .
\end{aligned}
$$

These are the sufficient conditions of Schur-concave of the generalized Muirhead means $M(p, q ; x, y)$ asserted by Theorem C.

\section{Conflict of Interests}

The authors declare that there is no conflict of interests regarding the publication of this paper.

\section{Acknowledgments}

This research was supported by the Natural Science Foundation of China under Grants 11171307 and 61374086, the Natural Science Foundation of Zhejiang Province under Grant LY13A010004, the Natural Science Foundation of Fujian province under Grant 2012J01014, and the Foundation of Scientific Research Project of Fujian Province Education Department under Grants JK2013051 and JK2012049.

\section{References}

[1] J. C. Kuang, Applied Inequalities, Shandong Science and Technology Press, Jinan, China, 4th edition, 2010.

[2] Q. J. Mao, "Dual means, logarithmic and Heronian dual means of two positive numbers," Journal of Suzhou College of Education, vol. 16, pp. 82-85, 1999.
[3] W. Janous, "A note on generalized Heronian means," Mathematical Inequalities \& Applications, vol. 4, no. 3, pp. 369-375, 2001.

[4] G. Jia and J. Cao, "A new upper bound of the logarithmic mean," Journal of Inequalities in Pure and Applied Mathematics, vol. 4, no. 4, article 80, 4 pages, 2003.

[5] D. M. Li, C. Gu, and H. N. Shi, "Schur convexity of the powertype generalization of Heronian means," Journal of Mathematics in Practice and Theory, vol. 6, no. 9, pp. 387-390, 2006.

[6] H. Shi, M. Bencze, S. Wu, and D. Li, "Schur convexity of generalized Heronian means involving two parameters," Journal of Inequalities and Applications, vol. 2008, Article ID 879273, 9 pages, 2008.

[7] L. L. Fu, B. Y. Xi, and H. M. Srivastava, "Schur-convexity of the generalized Heronian means involving two positive numbers," Taiwanese Journal of Mathematics, vol. 15, no. 6, pp. 2721-2731, 2011.

[8] Z. H. Yang, "Schur power convexity of the Daróczy means," Mathematical Inequalities \& Applications, vol. 16, no. 3, pp. 751762, 2013.

[9] T. Trif, "Monotonicity, comparison and Minkowski’s inequality for generalized MUIrhead means in two variables," Mathematica, vol. 48(71), no. 1, pp. 99-110, 2006.

[10] W. M. Gong, H. Shen, and Y. M. Chu, "The Schur convexity for the generalized Muirhead mean," Journal of Mathematical Inequalities. In press.

[11] W. Xia and Y. Chu, "The Schur multiplicative convexity of the generalized Muirhead mean values," International Journal of Functional Analysis, Operator Theory and Applications, vol. 1, no. 1, pp. 1-8, 2009.

[12] Y. M. Chu and W. F. Xia, "Necessary and sufficient conditions for the Schur harmonic convexity of the generalized Muirhead mean," Proceedings of A Razmadze Mathematical Institute, vol. 152, pp. 19-27, 2010.

[13] A. W. Marshall and I. Olkin, Inequalities: Theory of Majorization and Its Applications, vol. 143 of Mathematics in Science and Engineering, Academic Press, New York, NY, USA, 1979. 


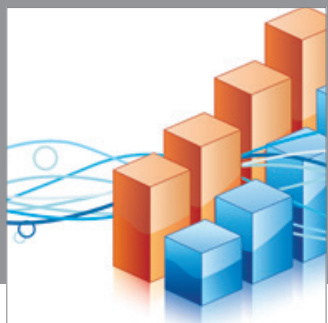

Advances in

Operations Research

mansans

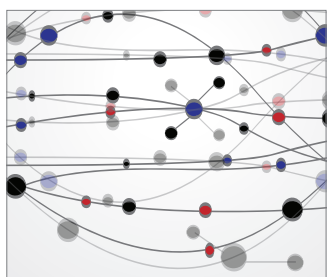

The Scientific World Journal
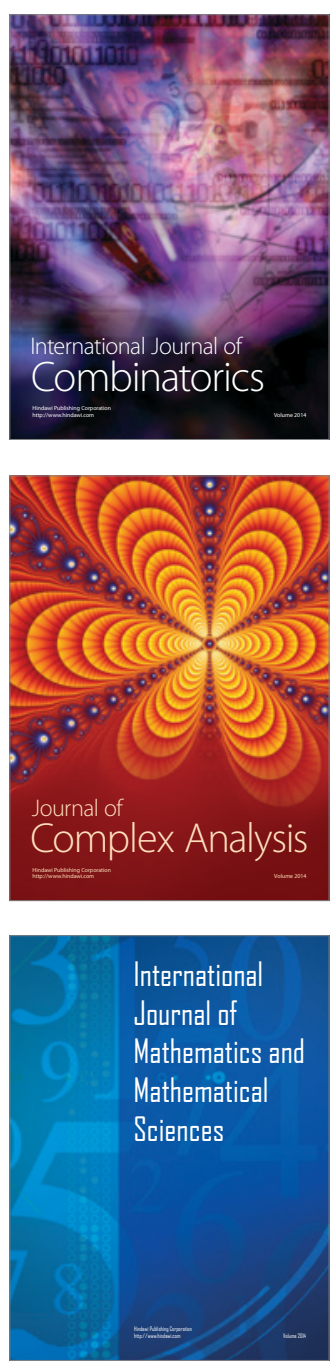
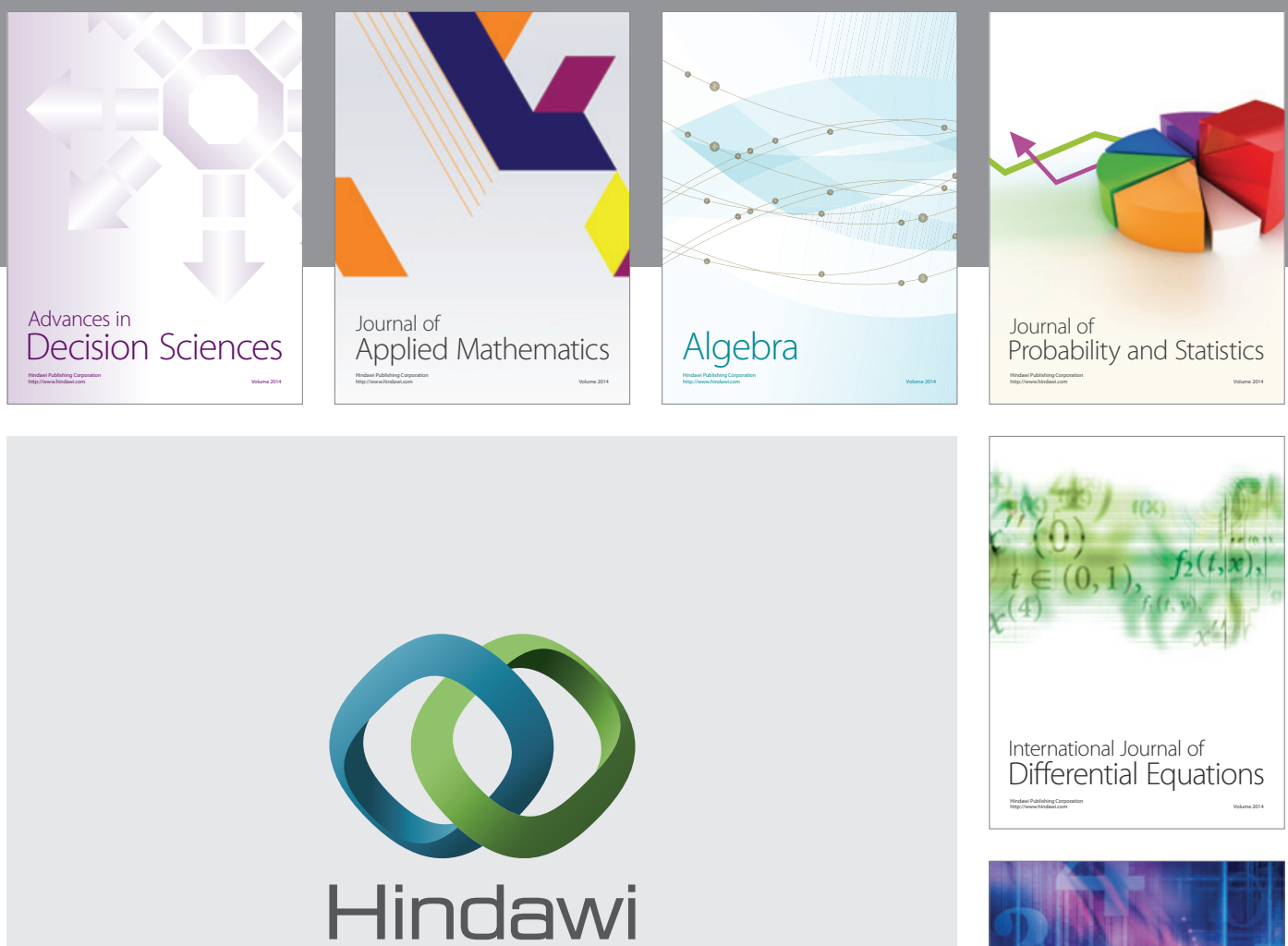

Submit your manuscripts at http://www.hindawi.com
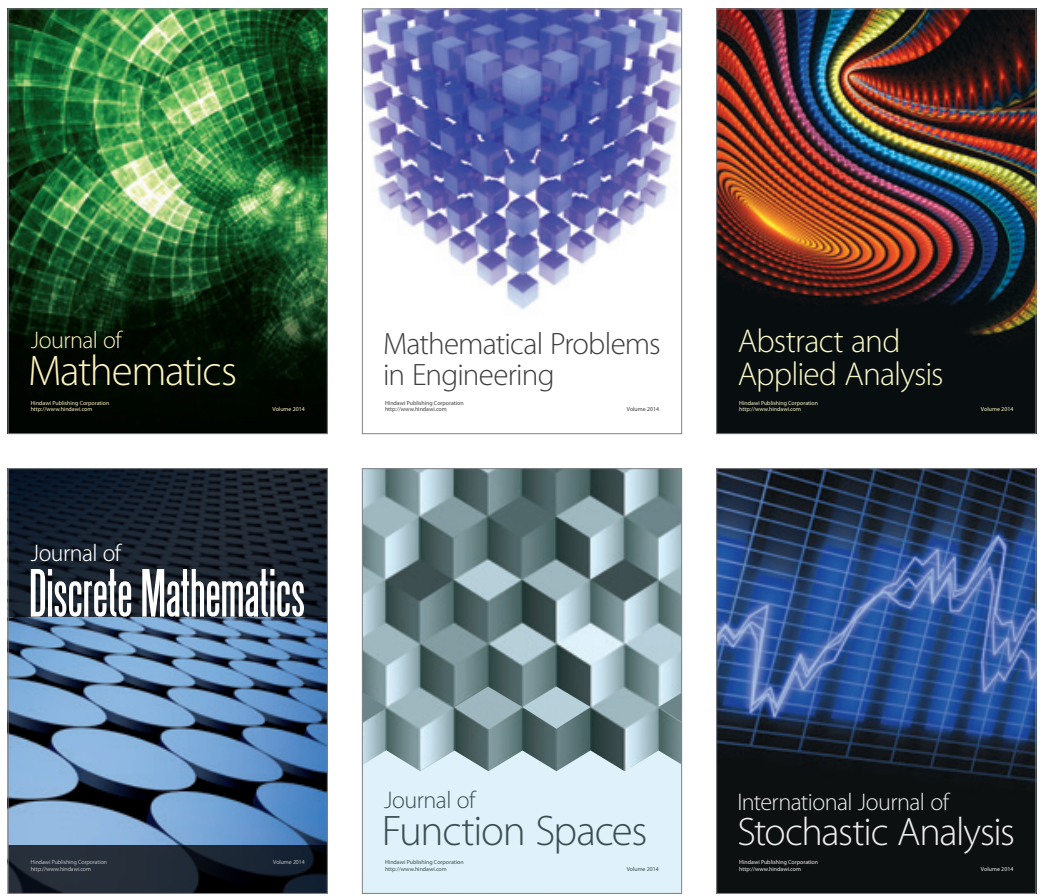

Journal of

Function Spaces

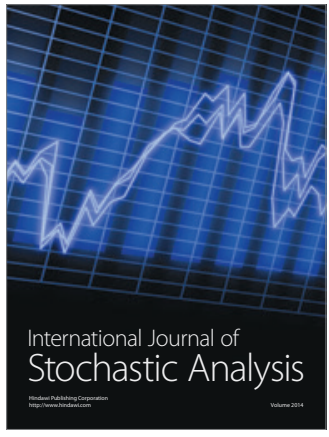

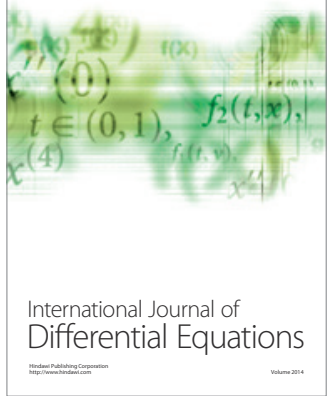
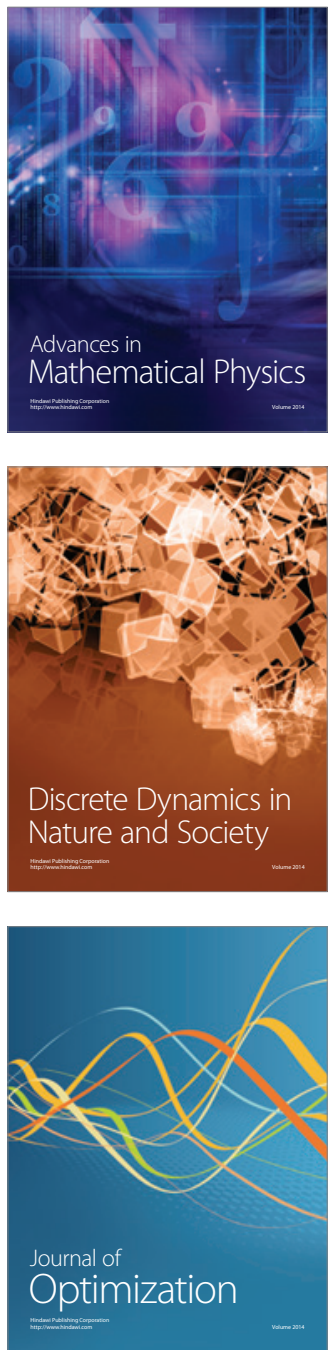